\title{
X-ray optics at NASA Marshall Space Flight Center
}

\author{
Stephen L. O’Dell ${ }^{\mathrm{a}}$, Carolyn Atkins ${ }^{\mathrm{b}}$, David M. Broadway ${ }^{\mathrm{a}}$, Ronald F. Elsner ${ }^{\mathrm{a}}$, \\ Jessica A. Gaskin ${ }^{\text {e }}$, Mikhail V. Gubarev ${ }^{\text {a }}$, Kiranmayee Kilaru ${ }^{\text {c }}$, Jeffery J. Kolodziejczak ${ }^{\mathrm{a}}$, \\ Brian D. Ramsey ${ }^{\text {a }}$, Jacqueline M. Roche ${ }^{a}$, Douglas A. Swartz ${ }^{\text {a }}$, Allyn F. Tennant ${ }^{\text {a }}$, \\ Martin C. Weisskopf ${ }^{a}$, and Vyacheslav E. Zavlin ${ }^{c}$ \\ ${ }^{a}$ NASA Marshall Space Flight Center, Huntsville, AL 35812, USA \\ ${ }^{\mathrm{b}}$ University of Alabama in Huntsville, Huntsville, AL 35899, USA \\ ${ }^{\mathrm{c}}$ Universities Space Research Association, Marshall Space Flight Center, \\ Huntsville, AL 35812, USA
}

\begin{abstract}
NASA's Marshall Space Flight Center (MSFC) engages in research, development, design, fabrication, coating, assembly, and testing of grazing-incidence optics (primarily) for x-ray telescope systems. Over the past two decades, MSFC has refined processes for electroformed-nickel replication of grazing-incidence optics, in order to produce highstrength, thin-walled, full-cylinder x-ray mirrors. In recent years, MSFC has used this technology to fabricate numerous x-ray mirror assemblies for several flight (balloon, rocket, and satellite) programs. Additionally, MSFC has demonstrated the suitability of this technology for ground-based laboratory applications-namely, $\mathrm{x}$-ray microscopes and cold-neutron microscopes and concentrators.

This mature technology enables the production, at moderately low cost, of reasonably lightweight $\mathrm{x}$-ray telescopes with good (15-30 arcsecond) angular resolution. However, achieving arcsecond imaging for a lightweight $\mathrm{x}$-ray telescope likely requires development of other technologies. Accordingly, MSFC is conducting a multi-faceted research program toward enabling cost-effective production of lightweight high-resolution x-ray mirror assemblies. Relevant research topics currently under investigation include differential deposition for post-fabrication figure correction, in-situ monitoring and control of coating stress, and direct fabrication of thin-walled full-cylinder grazing-incidence mirrors.
\end{abstract}

Keywords: X-ray telescopes, electroformed mirrors, x-ray optics, neutron optics, differential deposition, coating stress, optics fabrication

\section{INTRODUCTION}

Launched in 1999, the Chandra X-ray Observatory ${ }^{1,2}$ (Figure 1, Left) continues as NASA's flagship mission for x-ray astronomy. Its four nested, thick-walled, grazing-incidence mirror pairs (Figure 1, Right) uniquely provide subarcsecond x-ray imaging of cosmic sources. Currently, the US x-ray-astronomy community is considering mission concepts and enabling technologies for a worthy successor to Chandra. The optical performance requirements for this "X-ray Surveyor" are likely to call for an angular resolution comparable to that of Chandra, with an aperture area 30 times larger. ${ }^{3}$ Thus important technological and programmatic challenges ${ }^{4,5}$ lie in the fabrication, alignment, and mounting of lightweight grazing-incidence mirrors into a mirror assembly that affords sub-arcsecond x-ray imaging with an aperture area $\approx 3 \mathrm{~m}^{2}$.

The X-ray Astronomy Team at Marshall Space Flight Center (MSFC) began in 1977 with the arrival Martin Weisskopf to serve as Project Scientist for the Advanced X-ray Astrophysics Facility (AXAF), which became the Chandra X-ray Observatory. Since then, the primary responsibility of the Team has been to support of the Chandra Project during formulation, development, calibration, and operation of Chandra. We here briefly describe MSFC's in-house research and fabrication of grazing-incidence optics (primarily) for x-ray telescope systems.

\footnotetext{
* Contact author (SLO): stephen.l.odell@ nasa.gov; voice +1 (256) 961-7776; fax +1 (256) 961-7522

Postal address: NASA/MSFC/ZP12; 320 Sparkman Drive NW; Huntsville, AL 35805-1912 USA
} 

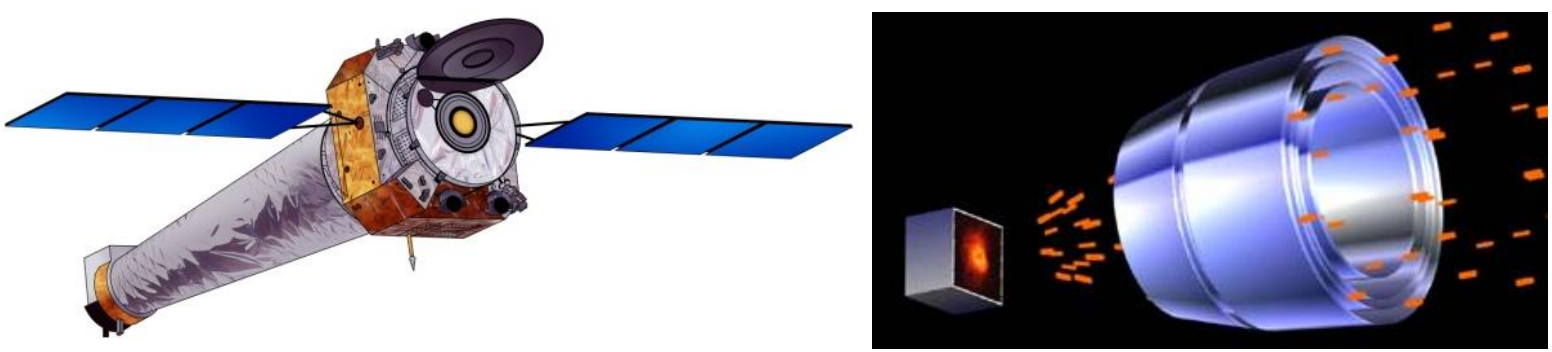

Figure 1. NASA's Chandra X-ray Observatory (Left) provides sub-arcsecond x-ray imaging of cosmic sources using four precision grazing-incidence mirror pairs (Right).

Over the past two decades, MSFC has refined processes for electroformed-nickel replication (ENR) of grazingincidence optics (\$2). Currently, MSFC uses this technology to produce (\$3) x-ray telescopes for sub-orbital (balloon and rocket) and in-space missions (\$3.1), as well as grazing-incidence optics for (ground-based) laboratory applications (§3.2). The angular resolution of ENR telescopes typically ranges from 15" to 30" half-energy width (HEW). While this angular resolution suffices for many applications, it does not approach the fine resolution of Chandra that is desired for an X-ray Surveyor. Consequently, MSFC is also conducting research (§4) toward the goal of lightweight sub-arcsecond $\mathrm{X}$-ray telescopes. Current research topics include post-fabrication correction of (full-shell and segmented) replica mirrors $(\S 4.1)$, control of coating stress $(\S 4.2)$, and direct fabrication of thin-walled full-shell mirrors $(\S 4.3)$.

\section{ELECTROFORMED-NICKEL REPLICATON (ENR)}

Replication copies the surface figure of a precision mandrel onto a complementary mirror. It has two advantages over direct fabrication (§4.3). First, as the mandrel can be thick-walled and very stiff, it is less susceptible to deformation during figuring and polishing. Second, as replication itself is inexpensive compared to precision figuring and polishing, it becomes much more cost-effective to use replicated mirrors if the design calls for several mirrors of the same size and shape. A disadvantage of replication is that the figure of the replica — especially if the mirror is very thin - does not perfectly match the shape of the precisely figured mandrel.

The prevalent replication technique for full-shell $\mathrm{x}$-ray mirrors is nickel electroforming, which is used for ESA's XMMNewton $^{6}$ and for several smaller satellite ${ }^{7,8,9,10,11}$ and sub-orbital ${ }^{12,13}$ missions. Pioneered for $\mathrm{X}$-ray optics in Prague ${ }^{14,15,16}$ and elsewhere ${ }^{17,18,19}$ and refined in Italy, ${ }^{20,21}$ MSFC has further improved the process over the past two decades. The production of replicated x-ray mirrors comprises two top-level procedures-mandrel fabrication $(\$ 2.1)$ and shell fabrication $(\$ 2.2)$.

\subsection{Mandrel fabrication}

Figure 2 outlines the basic steps in fabricating a precision mandrel for electroformed-nickel replication (ENR) of a fullshell grazing-incidence mirror. The mandrel typically incorporates primary $(\mathrm{P})$ and secondary $(\mathrm{S})$ conic frusta monolithically so that the $\mathrm{P}$ and $\mathrm{S}$ surfaces of a full-shell replica are consequentially coaxial. This feature and the mirror being a full shell greatly simplify alignment of mirror shells into a mirror assembly. While MSFC usually employs single-point diamond turning of the electroless nickel to figure the mandrel's $\mathrm{P}$ and $\mathrm{S}$ surfaces to the required optical prescriptions, other figuring processes - e.g., precision grinding - are possible and occasionally used. Final figuring, smoothing, and superpolishing of the mandrel's electroless-nickel surface utilize conventional lapping and polishing methods using a custom precision lathe. Of course, throughout the mandrel fabrication, precision metrology is essential: "The optic can be no better than the metrology." To this end, MSFC utilizes a full suite of metrology instrumentation spanning relevant spatial frequencies and geometries-including a large coordinate-measuring machine, circularity test stand, Fizeau interferometer, long-trace profilometer, optical surface profiler, and atomic-force microscope. 

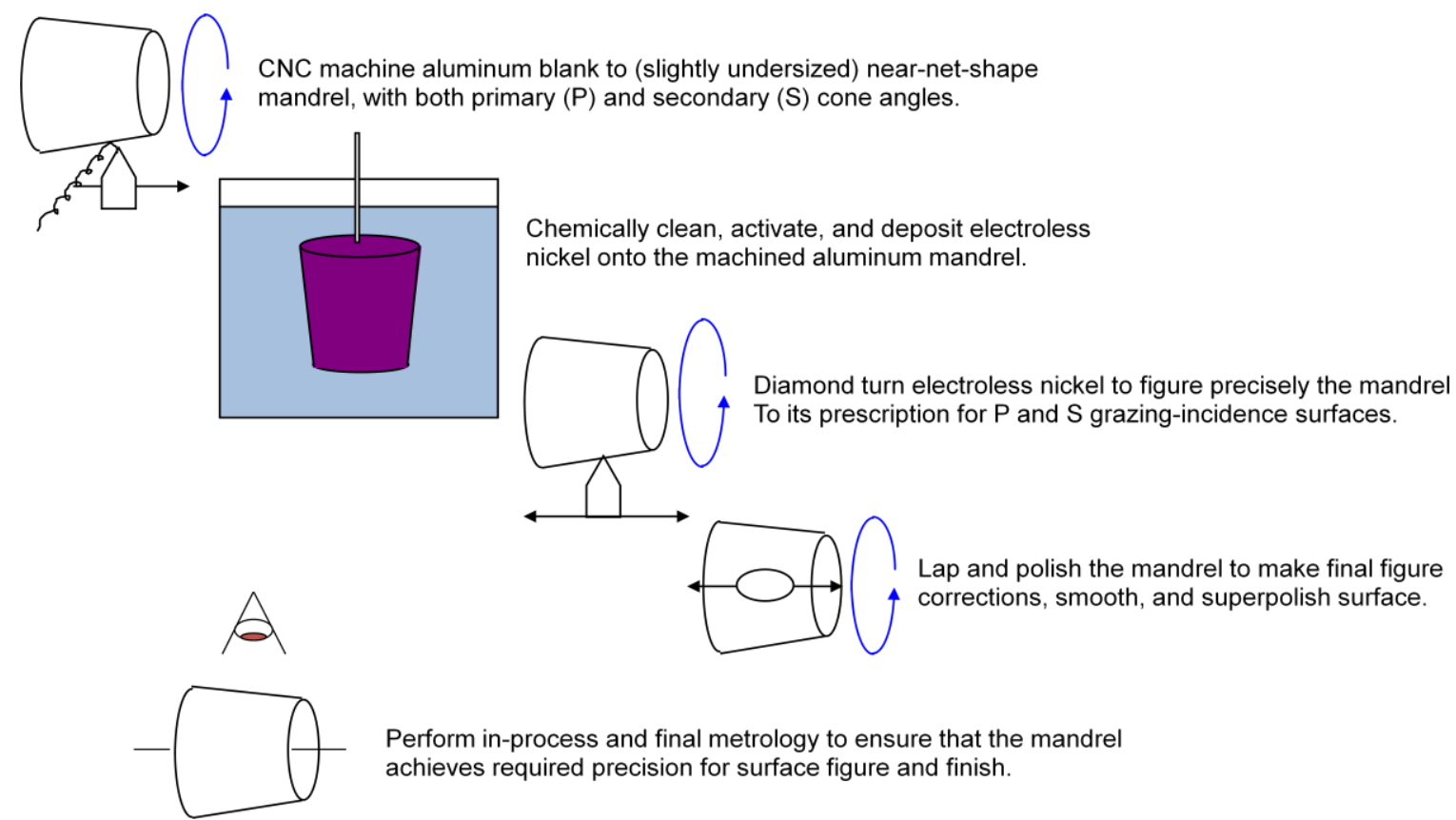

Perform in-process and final metrology to ensure that the mandrel achieves required precision for surface figure and finish.

Figure 2. Basic steps in fabricating a precision mandrel for electroformed nickel replication.

\subsection{Shell fabrication}

Figure 3 outlines the basic steps in electroforming a nickel replica full-shell grazing-incidence mirror off a precision electroless-nickel-plated aluminum mandrel (\$2.1). Again, the ENR mirrors usually are full shells and incorporate both (P and S) grazing-incidence surfaces required for true imaging. Over the past two decades, MSFC has developed several process refinements that now enable the successful fabrication of very thin $(\approx 100-\mu \mathrm{m})$ ENR mirror shells, although applications typically utilize thicker shells to reduce mount-induced distortions during alignment and assembly.

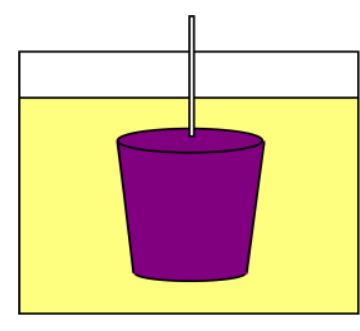

Ultrasonically clean and chemically passivate the electroless-nickel surface of the precision mandrel, in order to preserve surface finish and control adhesion.

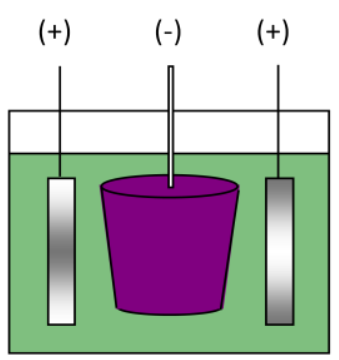

Electroform nickel or nickel-alloy shell onto the mandrel, with controlled uniform plating stress in order to ensure release with minimal distortion.

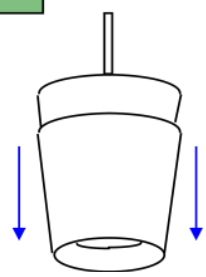

Separate shell from mandrel in a chilled-water bath; clean mandrel for additional replications; and deposit optical coating on inner surface of shell.

Figure 3. Basic steps in electroforming a nickel replica full-shell grazing-incidence mirror. 
One area of ENR process improvement is surface passivation - control of adhesion of the shell onto the mandrel. If adhesion is too low, the plating will not stick to the mandrel well enough to form a shell; if too high, the formed shell will not release in the chilled-water bath. ${ }^{\dagger}$ Historically, gold (deposited on the mandrel and transferred to the shell during replication) served both as a passivating layer and as the optical coating of the mirror. For most x-ray-astronomy applications, MSFC deposits an iridium optical coating onto the inner surface of the mirror shell after replication, in order to take advantage of its x-ray reflectance, which is somewhat higher than that of gold. To accomplish this, MSFC developed a chemical passivation process that allows electroforming nickel directly on an electroless-nickel plated surface. More recently, MSFC has collaborated with the Smithsonian Astrophysical Observatory (SAO), to demonstrate the use of a titanium nitride (TiN) as a durable passivation film on mandrels. ${ }^{22}$ Such a film is hard, negligibly increases the surface roughness, and enables deposition onto a mandrel of optical coatings (including multilayers) that adhere to and release with the shell. This approach is particularly useful for coating small-diameter full shells, which allow insufficient space for post-fabrication deposition of an optical coating.

A second area of ENR process improvement is control of electroforming stress and its uniformity. ${ }^{23}$ To avoid lifting the shell off the mandrel during electroforming, the deposition stress must be slightly tensile. In extreme instances, the deposition stress may be so tensile that the shell will not release upon cooling. Typically, the tensile stress is small and the shell separates; however, even a small residual tensile stress causes the ends of the mirror shell to hook inwards. If the electroforming stress is just slightly tensile but uniform, distortion by the residual stress is confined to a small region at the ends of the mirror shell and has little effect upon customary optical performance metrics-half-energy width (HEW) and full-with at half maximum (FWHM),

A third area of ENR process improvement is development of nickel-alloy platings that exhibit a much higher precision elastic limit (or micro-yield strength) than does pure nickel. Consequently, these nickel-alloy shells are significantly less susceptible than pure-nickel shells to plastic deformation during release and handling. With these and other process improvements, MSFC can produce very thin $(\approx 100-\mu \mathrm{m})$ ENR mirror shells of good (10-20 arcsecond) intrinsic angular resolution. Nevertheless, as thinner shells are more susceptible to mounting distortion, a general guideline is to make shells no thinner than the application requires-e.g., due to mass or filling-factor constraints.

\section{PRODUCTION OF ENR GRAZING-INCIDENCE OPTICS}

While MSFC is continuing research to improve further the performance of ENR grazing-incidence mirror systems, it has and is currently producing x-ray mirror systems for several (sub-orbital and space) x-ray telescopes (§3.1) and for (ground-based) laboratory applications $(\S 3.2)$.

\subsection{X-ray telescopes}

MSFC has produced numerous flight $\mathrm{x}$-ray mirror modules for several balloon, rocket, and satellite missions. In most cases, the telescope system comprises multiple ENR mirror assemblies and corresponding detectors, to benefit from the substantial cost savings in optics fabrication afforded by replication. Furthermore, for a given mass allocation, multiple mirror assemblies allow relatively stiffer mirrors than does a single mirror assembly with the same collecting area.

\subsubsection{High-Energy Replicated Optics to Explore the Sun (HEROES)}

HEROES is a joint balloon mission of MSFC and Goddard Space Flight Center (GSFC), designed to perform hard-xray $(25-75 \mathrm{keV})$ imaging of the sun (during the day) and of cosmic sources (during the night). Flown in 2013 September, the HEROES payload (Figure 4) includes 8 mirror assemblies with 6-m focal length. Each mirror assembly contains 14 coaxially nested iridium-coated ENR shells of 610-mm total $(\mathrm{P}+\mathrm{S})$ length and ranging in diameter from 50 $\mathrm{mm}$ to $94 \mathrm{~mm}$. The half-energy width of the mirror assemblies is typically HEW $\approx 30 \operatorname{arcsec}^{24}$

The HEROES payload is an enhanced version of MSFC's High-Energy Replicated Optics (HERO) payload, modified by GSFC to allow hard-x-ray observations of the sun. The original (proof-of-concept) HERO balloon flight in 2001 May obtained the first hard-x-ray focused images of cosmic sources, using just 2 modules of 3 ENR mirrors each. ${ }^{25,26}$

\footnotetext{
${ }^{\dagger}$ Release of the shell from the mandrel upon cooling relies upon the shell material (nickel or a nickel alloy) having a substantially lower coefficient of thermal expansion (CTE) than the predominant mandrel material (aluminum).
} 

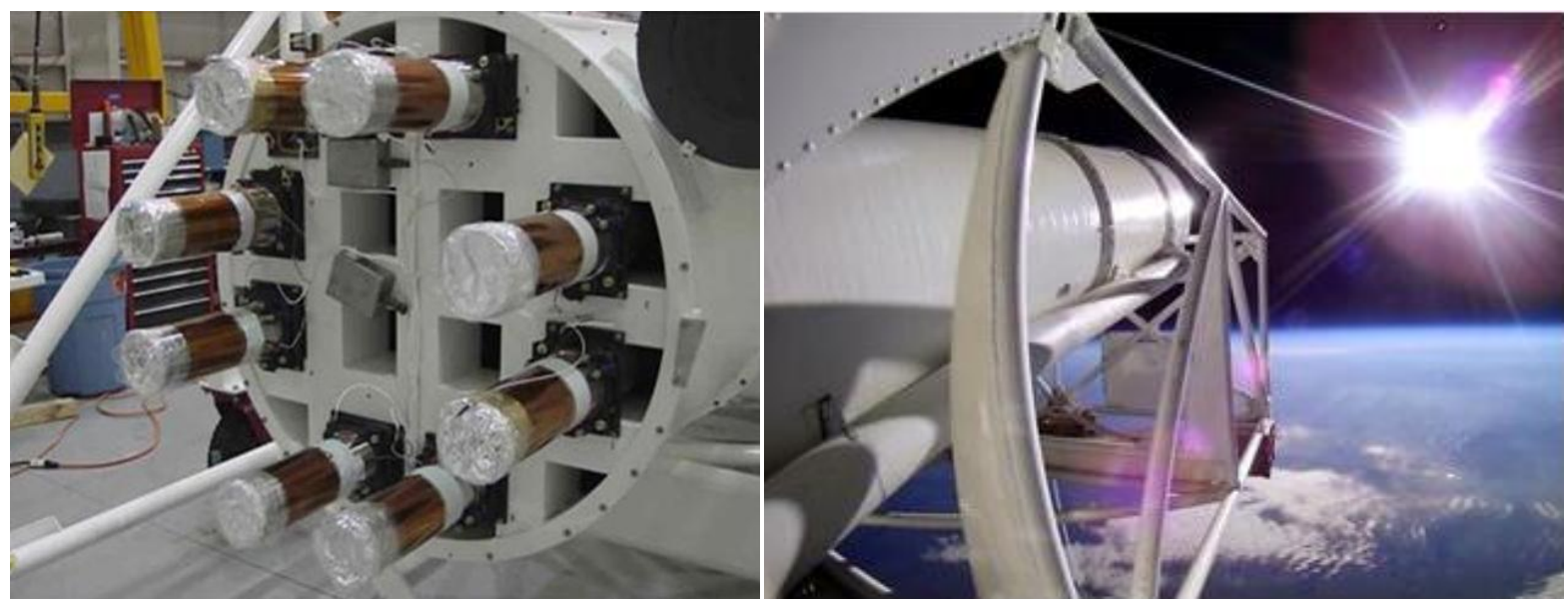

Figure 4. High-Energy Replicated Optics to Explore the Sun (HEROES), showing the telescope's 8 mirror assemblies (Left) and an in-flight picture of the telescope pointed toward the sun for heliospheric observations (Right).

\subsubsection{Focusing Optics X-ray Solar Imager (FOXSI)}

FOXSI is a sub-orbital rocket mission led by the University of California at Berkeley, using x-ray optics from MSFC and detectors from JAXA's Institute of Space and Astronautical Science. ${ }^{27}$ Designed to perform high-dynamic-range medium-energy $\mathrm{x}$-ray $(5-15 \mathrm{keV})$ imaging of the sun for the study of x-ray microflares, FOXSI has thus far had two successful flights-FOXSI-1 in 2012 November $^{28}$ and FOXSI-2 in 2014 December. The FOXSI telescope (Figure 5) includes 7 mirror assemblies with 2-m focal length. For FOXSI-1 (FOXSI-2), each mirror assembly contains 7 (10) coaxially nested iridium-coated ENR shells of 600-mm P+S length and ranging in diameter from 76 (63) $\mathrm{mm}$ to 103 $\mathrm{mm}$. The mirror assemblies exhibit an imaging performance with HEW $\approx 25 \operatorname{arcsec}$ and FWHM $\approx 5 \operatorname{arcsec}$, the later metric being more important for this application.
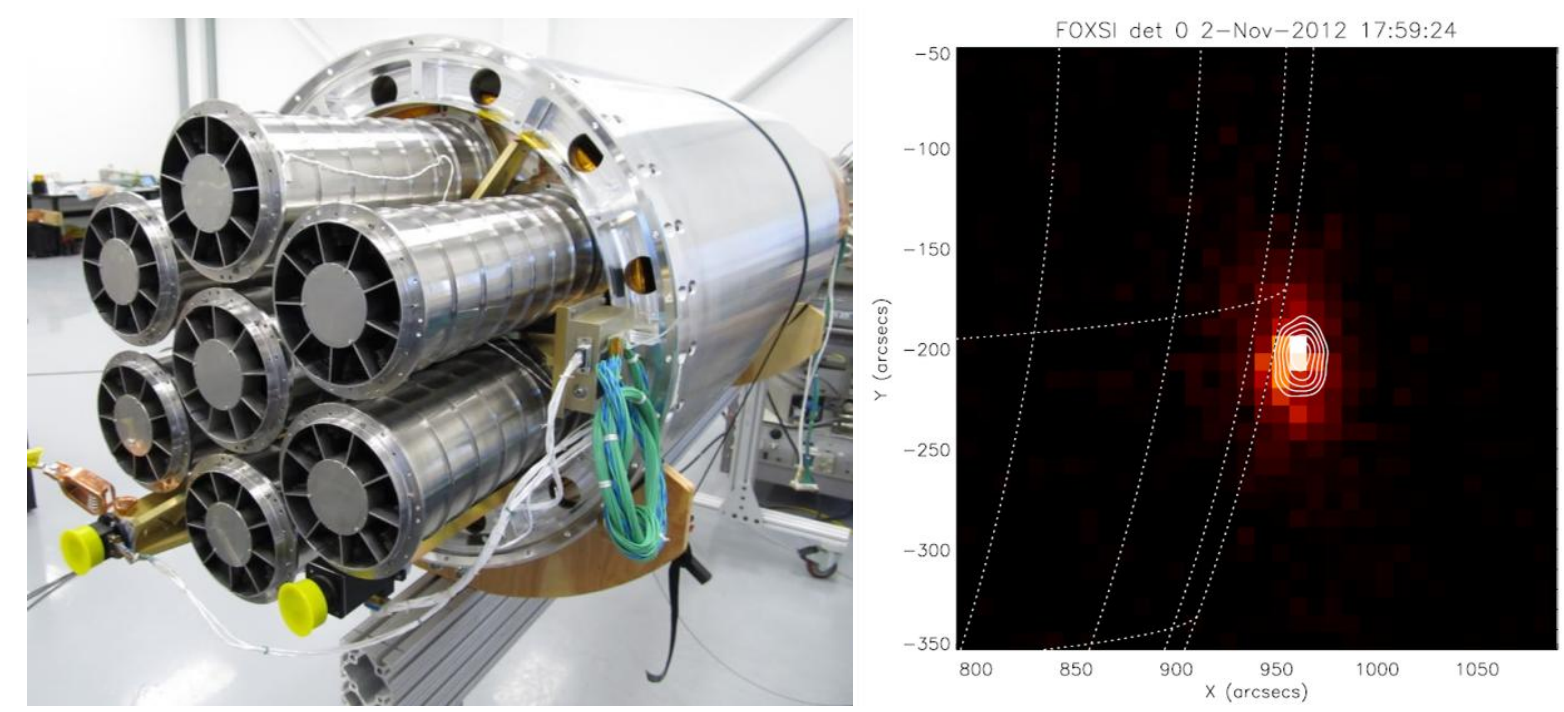

Figure 5. Focusing Optics X-ray Solar Imager (FOXSI), showing the telescope's 7 mirror assemblies (Left) and an image of a solar microflare (Right) obtained during the first FOXSI flight.

\subsubsection{Micro-X}

Micro-X is a sub-orbital rocket mission led by the Massachusetts Institute of Technology (MIT), designed to obtain soft-x-ray $(0.2-3 \mathrm{keV})$ non-dispersive high-spectral-resolution imaging of supernova remnants. ${ }^{29}$ Built in collaboration with Goddard Space Flight Center (GSFC) and the National Institute of Standards and Technology (NIST), the detector 
is a micro-calorimeter pixilated array. While the initial Micro-X flight(s) will employ aluminum-foil segmented $\mathrm{x}$-ray mirrors from GSFC ${ }^{30}$ MIT plans to utilize ENR full-shell x-ray optics from MSFC on a later flight (2017 or later). The ENR telescope (Figure 6) comprises a single mirror assembly with $2.5-\mathrm{m}$ focal length. The Micro-X ENR mirror assembly will contain 5 coaxially nested rhodium-coated ENR shells of $600-\mathrm{mm} \mathrm{P+S}$ length and ranging in diameter from $383 \mathrm{~mm}$ to $444 \mathrm{~mm}$, with a required angular resolution HEW < 30 arcsec.

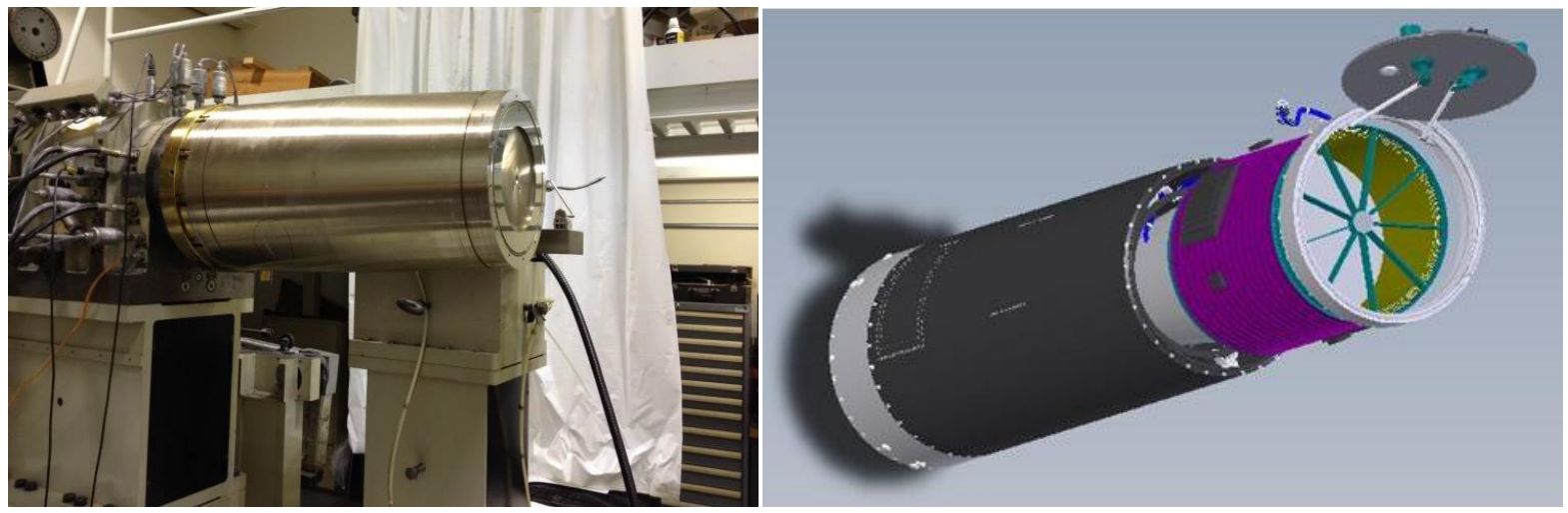

Figure 6. Micro-X ENR x-ray optics, showing fabrication of the first mandrel (Left) and a schematic of the optical bench (Right).

\subsubsection{Astronomical Röntgen Telescope (ART) on Spectrum-Röntgen-Gamma (SRG)}

Led by the Space Research Institute (IKI) of the Russian Academy of Sciences, the SRG satellite ${ }^{31}$ is to be launched into an L2 (outer Lagrange point) halo orbit in late 2016. SRG will conduct an x-ray all-sky survey during its first 4 years, using two complementary arrays of x-ray telescopes. The primary telescope array is the Extended RÖntgen Survey with an Imaging Telescope Array (eROSITA), ${ }^{32}$ led by the Max-Planck-Institut für extraterrestrische Physik (MPE). eROSITA is a soft-x-ray $(0.3-10 \mathrm{keV})$ imaging system comprising 7 mirror assemblies with 1.6-m focal length, each with a pn-CCD detector. ${ }^{33}$ Each eROSITA mirror assembly comprises 54 coaxially nested gold-coated ENR full-shell mirrors, produced by Media Lario (Italy). ${ }^{34}$
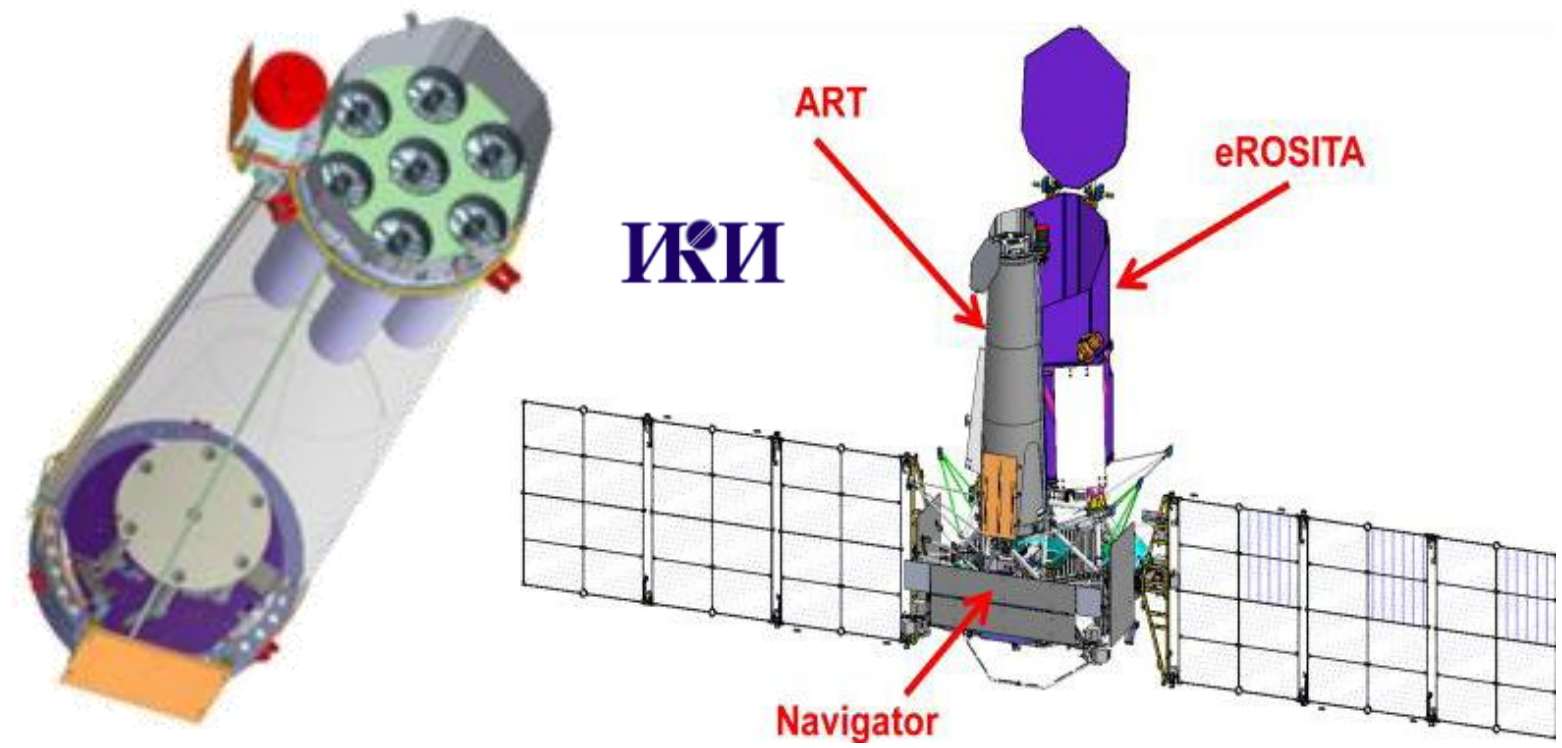

Figure 7. Astronomical Röntgen Telescope (ART) onboard Spectrum-Röntgen-Gamma (SRG). The ART drawing (Left) shows its 7 mirror assemblies and star tracker (top) and 7 detector collimators (bottom). The SRG drawing (Right) displays the two co-aligned telescope optical benches for ART and for eROSITA. 
Complementary to eROSITA, ART ${ }^{35}$ is a medium-energy x-ray $(5-25 \mathrm{keV})$ imaging system comprising 7 MSFCprovided mirror assemblies with $2.7-\mathrm{m}$ focal length ${ }^{36}$ each with an IKI-provided cadmium-telluride (CdTe) detector with crossed-strip-electrode read-out. ${ }^{37}$ Each ART mirror assembly comprises 28 coaxially nested iridium-coated ENR full-shell mirrors of 580-mm P+S length and ranging in diameter from $49 \mathrm{~mm}$ to $145 \mathrm{~mm}$. MSFC has completed the ground calibration of the flight mirror assemblies ${ }^{38}$ and delivered them to IKI. The typical imaging performance of the ART mirror assemblies is HEW $\approx 25$ arcsec on axis at best focus. However, to optimize sensitivity for the all-sky survey, the detector plane will likely be displaced intra-focal, giving HEW $<40$ arcsec over a 20 -arcmin field of view.

\subsection{Ground-based applications}

Although production of x-ray telescopes for high-energy astrophysics is the primary motivation for MSFC's development and fabrication of ENR grazing-incidence optics, the technology and supporting infrastructure are relevant to several ground-based applications. Space applications are usually more demanding than laboratory applications, for which mass constraints and collecting-area requirements are not an issue. Thus, grazing-incidence optics for laboratory applications are comparatively smaller with thicker walls, making them less susceptible to induced (mounting) distortions.

MSFC is exploring collaborations to utilize ENR grazing-incidence optics for laboratory applications. Two such applications are small-animal radionuclide imaging (\$3.2.1) and cold-neutron imaging (\$3.2.2).

\subsubsection{Small-animal radionuclide imaging}

Originally funded by the US National Institutes of Health, MSFC has collaborated with Lawrence Livermore National Laboratory, Smithsonian Astrophysical Observatory (SAO), and University of California in San Francisco to explore the use of ENR grazing-incidence mirrors for radionuclide imaging of small animals. ${ }^{39}$ About a tenth the size of ENR mirror shells used for $\mathrm{x}$-ray telescopes, the microscope mirror shells (Figure 8) have a 480-mm focal length, 60-mm total $\mathrm{P}+\mathrm{S}$ length, 60-70-mm diameters, and multilayer coating (by SAO). The demonstration design employed 4 coaxially nested shells configured to provide $4 \mathrm{X}$ magnification radionuclide imaging using iodine ${ }^{125} \mathrm{I}(27 \mathrm{keV})$ or technetium ${ }^{99 \mathrm{~m}} \mathrm{Tc}(18 \mathrm{keV})$. The imaging performance for this design is currently FWHM $\approx 100 \mu \mathrm{m}$.
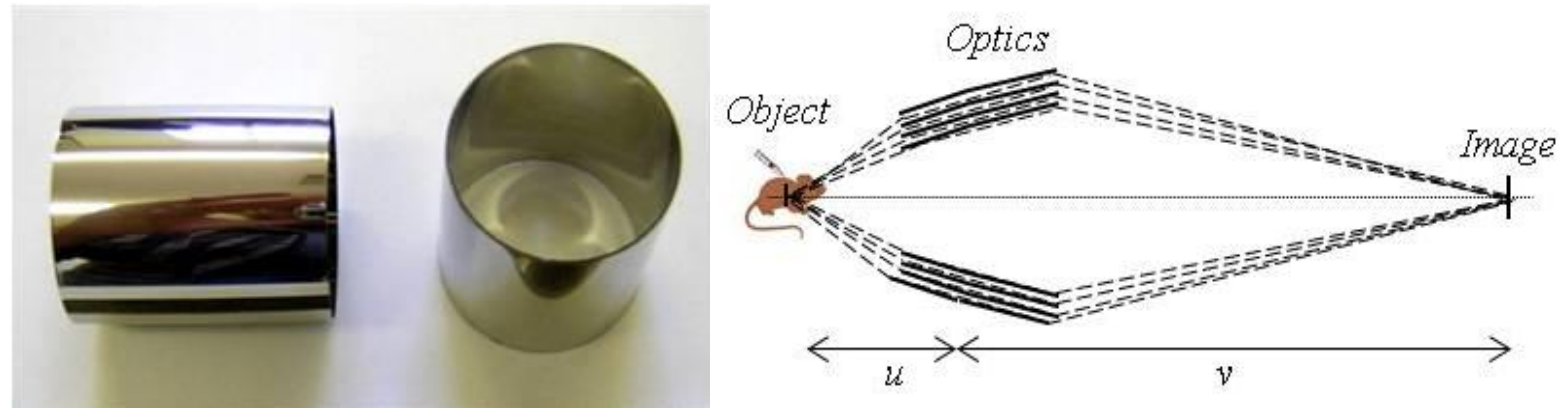

Figure 8. X-ray microscope for radionuclide imaging, showing 2 of 4 hyperboloid-ellipsoid shells (Left) coaxially nested to provide $4 \mathrm{X}$ magnification (Left).

\subsubsection{Cold-neutron imaging}

Cold neutrons (of order $10 \mathrm{~K}$ or $1 \mathrm{meV}$ ) have deBroglie wavelengths comparable to $\mathrm{x}$-ray (around $1 \mathrm{keV}$ ) wavelengths and reflect efficiently off smooth pure nickel surfaces. Hence, electroformed pure-nickel replicated grazing-incidence mirrors will image cold neutrons. In collaboration with the Massachusetts Institute of Technology (MIT) and the Oak Ridge National Laboratory, MSFC built and demonstrated a cold-neutron microscope (Figure 9). ${ }^{40,41}$ This neutron microscope is essentially identical to the $\mathrm{x}$-ray microscope for radionuclide imaging ( $\$ 3.2 .1$ and Figure 8 ), except that it uses uncoated pure-nickel ENR mirrors, as opposed to multilayer-coated nickel-alloy ENR mirrors. ${ }^{*}$

\footnotetext{
${ }^{*}$ Multilayer coatings containing nickel—such as those composed of nickel/titanium bilayers—-may be used to extend the energy range for neutron imaging, much as they are used to do so for hard x-rays.
} 


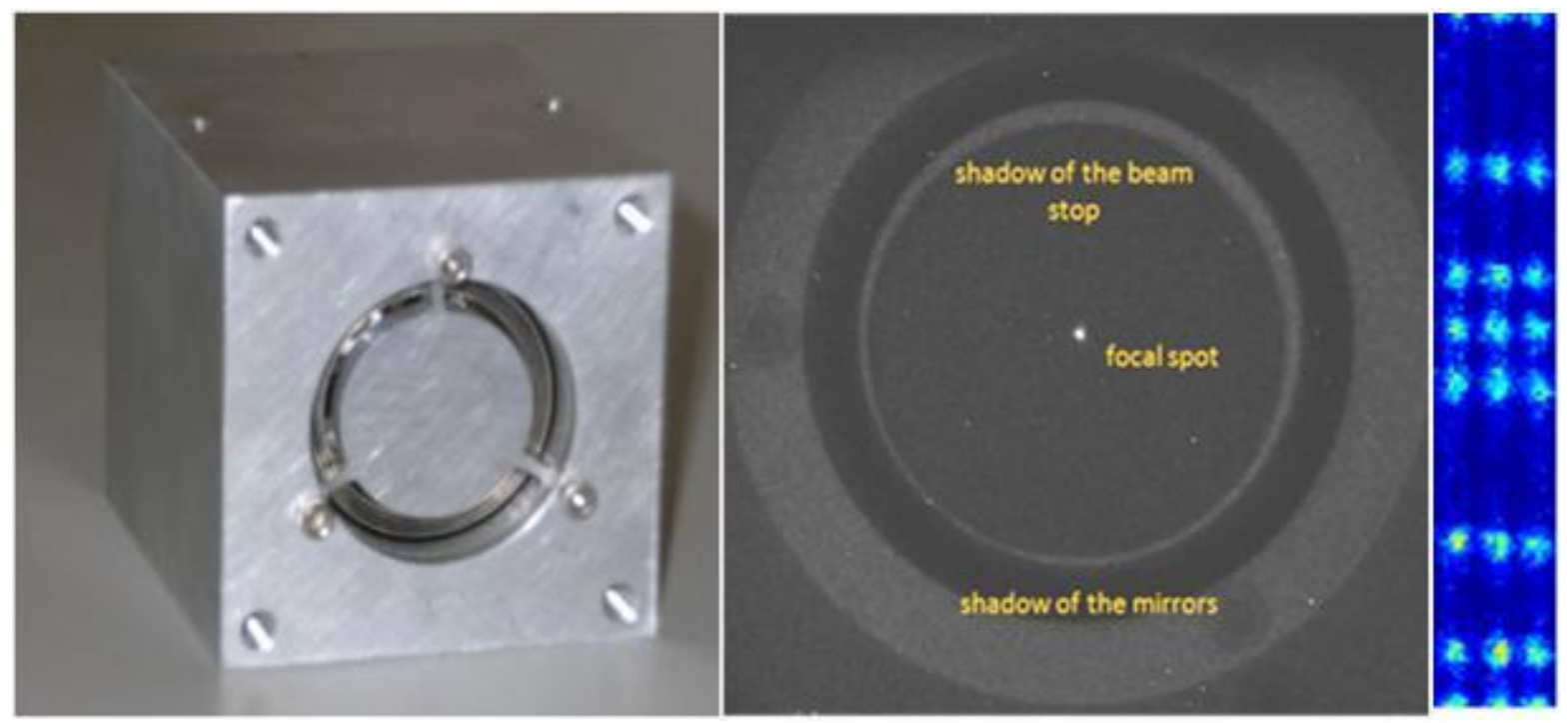

Figure 9. A demonstration neutron microscope (Left) that images cold neutrons (Center). The cold-neutron microscope achieved a resolution FWHM $=70 \mu \mathrm{m}$, as evidenced in a neutron image (Right) of an array of 100- $\mu \mathrm{m}$-diameter pinholes at 200- $\mu$ m pitch.

In addition to providing focused imaging, neutron optics also concentrate the intensity of cold-neutron source for smallangle neutron-scattering analyses of materials. Placed in a neutron reactor beam, even a small grazing-incidence mirror system (Figure 9) can concentrate the neutron intensity by factors of 50-100 into a small volume. ${ }^{42,43}$
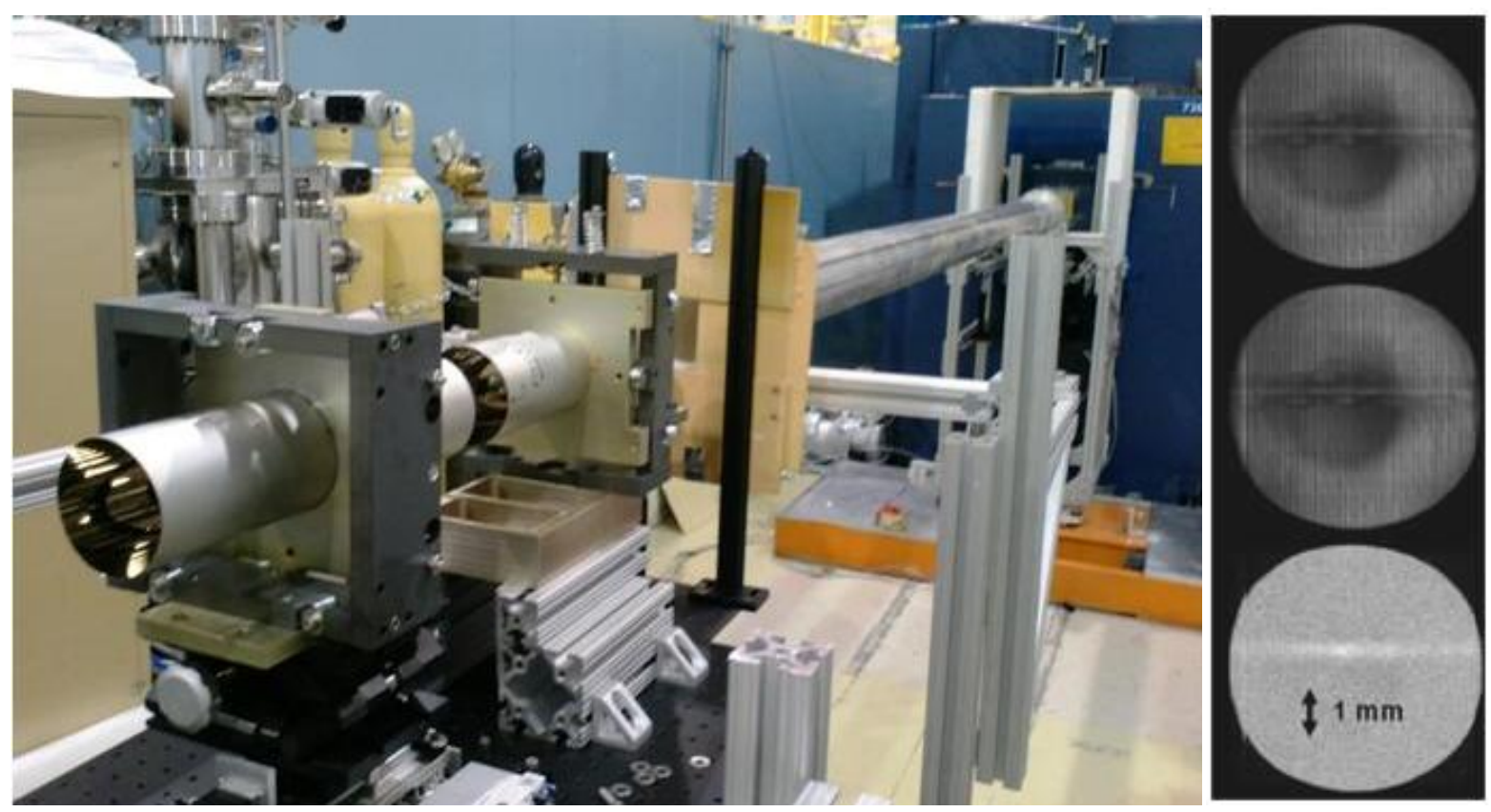

Figure 10. A neutron beam line at NIST (Left) for demonstration of cold-neutron imaging for potential use in a permanent beam line for diverse research applications. One such applied-research use is to measure the accumulation of water on a fuel-cell electrode (Right). 
Currently, MSFC is funded by NIST to develop grazing-incidence neutron optics for a multi-step demonstration: (1) High-resolution imaging; (2) 1X-magnification imaging; and (3) large-magnification imaging. Such a neutron-optics beam line would be used for diverse studies in basic and applied sciences and engineering (Figure 10).

\section{RESEARCH TOWARD HIGH-RESOLUTION X-RAY OPTICS}

MSFC's development and production of ENR grazing-incidence mirror systems successfully support numerous space and laboratory applications $(\S 3)$ requiring good (HEW $=10-30 \mathrm{arcsec})$ angular resolution. However, even with perfect mandrels, residual stress in the replication process and mount-induced distortions may limit the inherent angular resolution of thin replicated X-ray optics to HEW > 5 arcsec. Achieving sub-arcsecond imaging - as needed for the Xray Surveyor-will likely require a substantially modified or a totally different approach. Accordingly, MSFC is engaged in research that may contribute to achieving a large-area sub-arcsecond x-ray telescope. Here we briefly describe three such research topics.

\subsection{Differential deposition}

Fabrication errors or mount-induced distortions may need to be corrected if the desired angular resolution of an optic is to be attained. For thin mirrors, the challenge is to correct such figure errors with minimal force and, if possible, to do so in situ. Instead of removing material to correct the surface figure of a mirror, differential deposition ${ }^{44,45,46}$ adds material—effectively filling in valleys rather than abrading the hills (Figure 11).

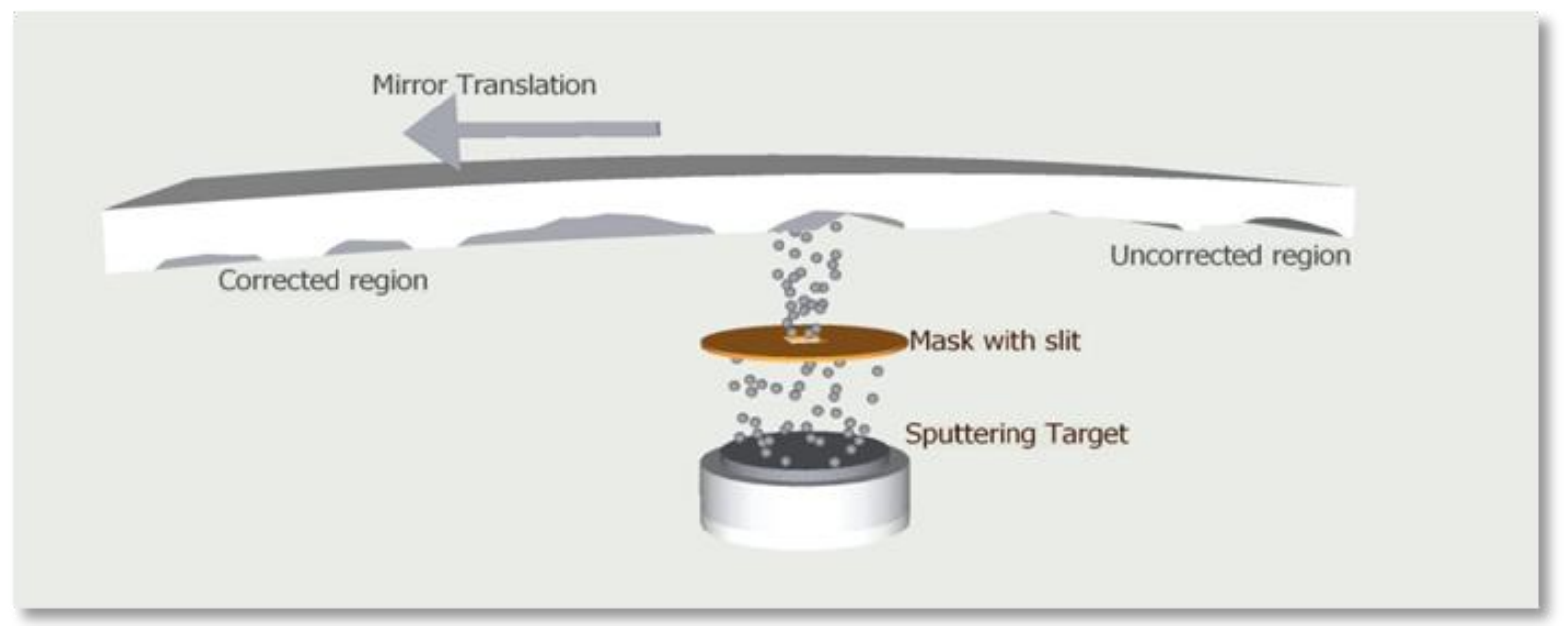

Figure 11. Differential deposition to correct figure errors on a mirror's surface.

MSFC is developing this process ${ }^{47,48}$ to correct the surface figure of light-weight grazing-incidence mirrors. Based upon metrology during a sequence of differential-deposition runs, Figure 12 shows that the process can indeed improve the surface figure of a grazing-incidence mirror. Note that the half-power diameter (HPD = HEW, Right panel) is a tworeflection equivalent calculated from the axial-profile metrology (Left panel) without and with filtering on spatial frequency. X-ray tests are planned for later this year.

\subsection{Control of coating stress}

Coating stress deforms a thin mirror due to a film-on-substrate bimorph-like effect, as calculated using the Stoney equation. Hence, it is important to control and to minimize coating stress and to distinguish intrinsic coating stress from thermally induced stresses due to film and substrate having different coefficients of thermal expansion (CTE). Accordingly, MSFC is engaged in a detailed research program to measure and to control coating stress, while depositing high-quality films - low surface roughness and good adhesion. ${ }^{49}$ An early product of this research is the development of an in situ stress monitor to measure coating stress during deposition of the film. ${ }^{50}$ 

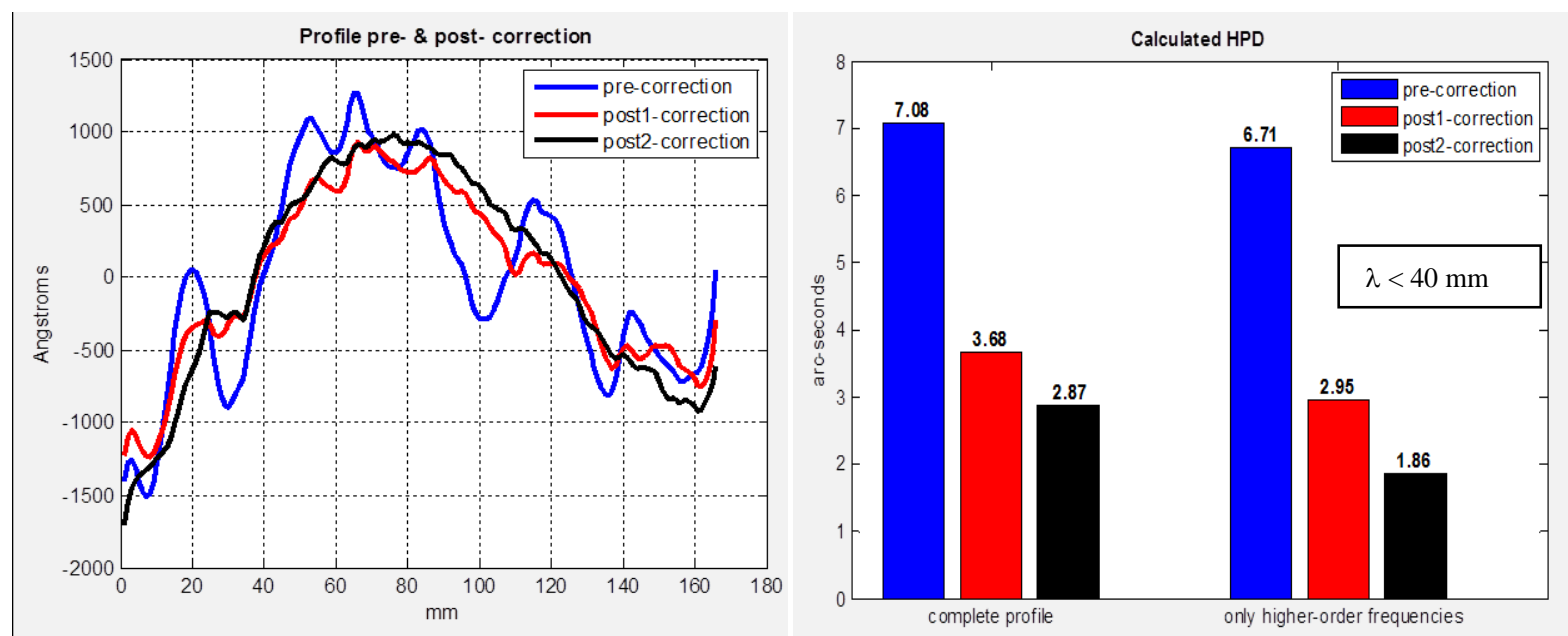

Figure 12. Metrology results showing the axial profile (Left) before correction and after the first and second corrections using differential deposition, as well as the corresponding calculated HEW (Left).

\subsection{Direct fabrication}

The remarkable Chandra mirrors were fabricated to sub-arcsecond precision using conventional direct-i.e., nonreplication-fabrication and very accurate metrology on thick $(\approx 20 \mathrm{~mm})$ Zerodur ${ }^{\mathrm{TM}}$ full shells. The Osservatorio Astronomico di Brera ${ }^{51,52}$ and, more recently, $\mathrm{MSFC}^{53}$ are investigating direct fabrication of full-cylinder x-ray mirrors that are nearly an order of magnitude thinner than the Chandra mirrors. For its research into direct fabrication of thinwalled full-shell grazing-incidence mirrors, MSFC is utilizing (Figure 13) a Zeeko ${ }^{\mathrm{TM}}$ Intelligent Robotic Polisher (IRP) for 7-axis CNC optical figuring and polishing, along with MSFC metrology instrumentation (§2.1).

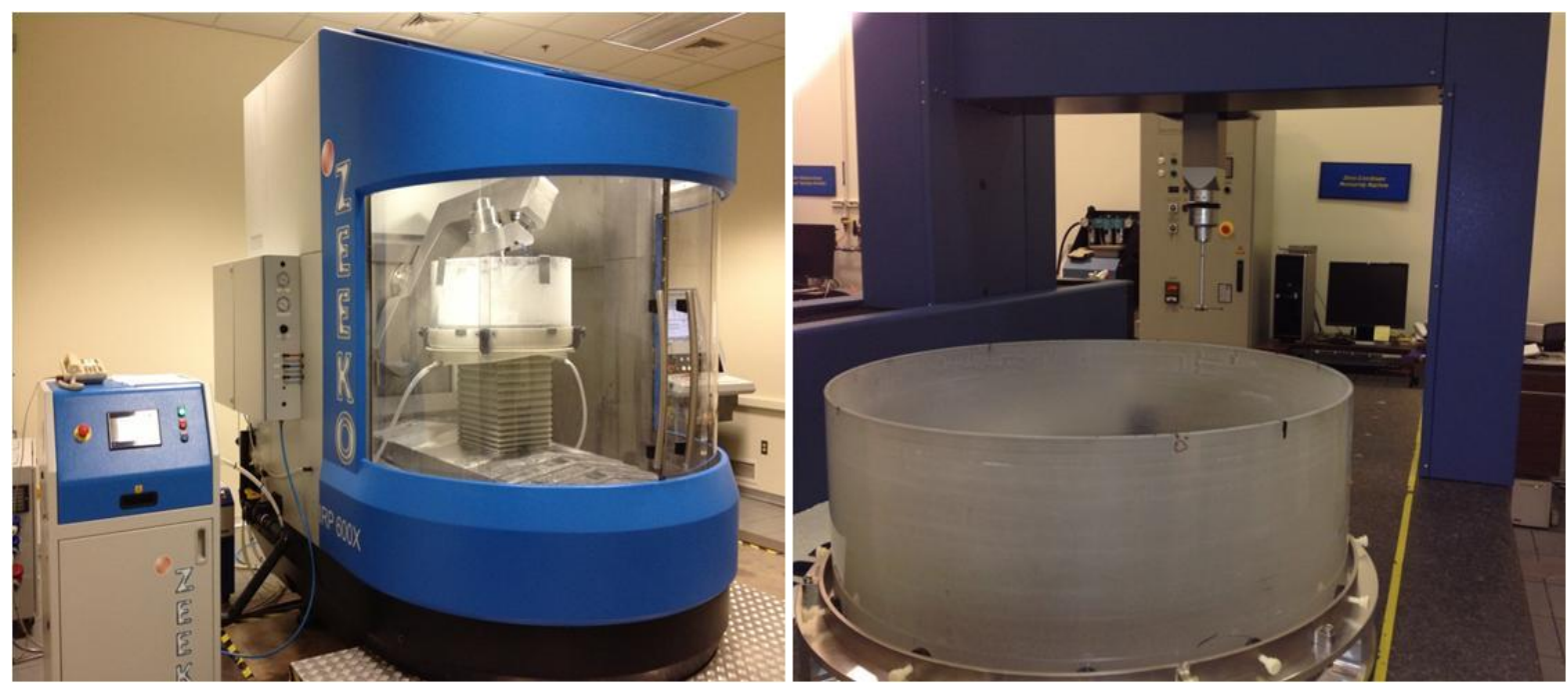

Figure 13. Zeeko Intelligent Robotic Polisher (IRP, Left) and Zeiss Coordinate Measuring Machine (CMM, Right), each shown with a 500-mm-diameter thin-walled full shell. 


\section{ACKNOWLEDGMENTS}

We gratefully acknowledge numerous grants and contracts from NASA and other agencies - as well as MSFC internal funding - that have supported our research and development of grazing-incidence optics over the past two decades. We also thank our collaborators for fruitful partnerships and for some images and drawings used in this article.

\section{REFERENCES}

1 O'Dell, S. L., \& Weisskopf, M. C., "The role of project science in the Chandra X-ray Observatory”, SPIE 6271, 07 14pp (2006).

2 Weisskopf, M. C., Tananbaum, H. D., Van Speybroeck, L. P., \& O'Dell, S. L., "Chandra X-ray Observatory (CXO): overview”, SPIE 4012, 2-16 (2000).

3 Weisskopf, M. C., Gaskin, J. A., Tananbaum, H. D., \& Vikhlinin, A., "Beyond Chandra - the X-ray Surveyor", SPIE 9510, these proceedings (2015).

4 O'Dell, S. L., Aldcroft, T. L., Allured, R., Atkins, C., Burrows, D. N., Cao, J., Chalifoux, B. D., Chan, K.-W., Cotroneo, V., Elsner, R. F., Graham, M. E., Gubarev, M. V., Heilmann, R. K., Johnson-Wilke, R. L., Kilaru, K., Kolodziejczak, J. J., Lillie, C. F., McMuldroch, S., Ramsey, B. D., Reid, P. B., Riveros, R. E., Roche, J. M., Saha, T. T., Schattenburg, M. L., Schwartz, D. A., Trolier-McKinstry, S. E., Ulmer, M. P., Vaynman, S., Vikhlinin, A., Wang, X., Weisskopf, M. C., Wilke, R. H. T., \& Zhang, W. W., "Toward large-area sub-arcsecond x-ray telescopes", SPIE 9208, 05 14pp (2014).

5 O'Dell, S. L., Brissenden, R. J., Davis, W. N., Elsner, R. F., Elvis, M. S., Freeman, M. D., Gaetz, T., Gorenstein, P., Gubarev, M. V., Jerius, D., Juda, M., Kolodziejczak, J. J., Murray, S. S., Petre, R., Podgorski, W., Ramsey, B. D., Reid, P. B., Saha, T., Schwartz, D. A., Trolier-McKinstry, S., Weisskopf, M. C., Wilke, R. H. T., Wolk, S., \& Zhang, W. W., "High-resolution x-ray telescopes", SPIE 7803, 0H 19pp (2010).

6 de Chambure, D., Laine, R., van Katwijk, K., Kletzkine, P., Valenzuela, A., Grisoni, G., Canali, M., Hofer, S., Tock, J. P., Domken, I., Stockman, Y., Hansen, H., Leonard, M., Aschenbach, B., \& Braeuninger, H. W., "Lessons learned from the development of the XMM optics", SPIE 3737, 2-17 (1999).

7 Conti, G., Chiappetti, L., Molendi, S., Sacco, B., Cusumano, G., La Rosa, G., Maccarone, M. C., Mineo, T., Boella, G., \& Citterio, O., "In-flight performances of grazing incidence x-ray optics on board the x-ray astronomy satellite BeppoSAX”, SPIE 3113, 394-403 (1997).

8 Burrows, D. N., Hill, J. E., Nousek, J. A., Wells, A. A., Chincarini, G., Abbey, A. F., Beardmore, A. P., Bosworth, J., Br\&auml;uninger, H. W., Burkert, W., Campana, S., Capalbi, M., Chang, W., Citterio, O., Freyberg, M. J., Giommi, P., Hartner, G. D., Killough, R., Kittle, B., Klar, R., Mangels, C., McMeekin, M., Miles, B. J., Moretti, A., Mori, K., Morris, D. C., Mukerjee, K., Osborne, J. P., Short, A. D. T., Tagliaferri, G., Tamburelli, F., Watson, D. J., Willingale, R., \& Zugger, M. E., "The Swift X-Ray Telescope”, SPIE 5165, 201-216 (2004).

9 Friedrich, P., Bräuninger, H., Budau, B., Burkert, W., Burwitz, V., Dennerl, K., Eder, J., Freyberg, M., Gaida, R., Hartner, G., Menz, B., Pfeffermann, E., Predehl, P., Rohé, C., \& Schreib, R., "Development and testing of the eROSITA mirror modules", SPIE 8443, 1S 8pp (2012).

10 Burwitz, V., Friedrich, P., Bräuninger, H., Budau, B., Burkert, W., Eder, J., Freyberg, M., Hartner, G., Pfeffermann, E., Predehl, P., Arcangeli, L., Borghi, G., Borroni, A., Citterio, O., Ferrario, I., Grisoni, G., Marioni, F., Ritucci, A., Rossi, M., Valsecchi, G., \& Vernani, D., "Development and testing of the eROSITA mirror modules", SPIE 8147, 08 6pp (2011).

11 Gubarev, M., Ramsey, B., O'Dell, S. L., Elsner, R., Kilaru, K., McCracken, J., Pavlinsky, M., Tkachenko, A., Lapshov, I., Atkins, C., \& Zavlin, V., "Development of mirror modules for the ART-XC instrument aboard the Spectrum-Roentgen-Gamma mission", SPIE 8861, 0K 6pp (2013).

12 Atkins, C., Ramsey, B., Kilaru, K., Gubarev, M., O'Dell, S., Elsner, R., Swartz, D., Gaskin, J., \& Weisskopf, M., "X-ray optic developments at NASA's MSFC", SPIE 8777, 0W 9pp (2013). 
13 Ramsey, B. D., Elsner, R. F., Engelhaupt, D., Gubarev, M. V., Kolodziejczak, J. J., O'Dell, S. L., Speegle, C. O., \& Weisskopf, M. C., "The development of hard x-ray optics at MSFC”, SPIE 5168, 129-135 (2004).

14 Hudec, R., Valníček, B., Pražák, V., \& Šolc, I., "Properties of galvanoplastic grazing incidence mirrors for astronomical applications”, BAICz 32, 188-191 (1981).

15 Hudec, R., \& Valníček, B., “Czechoslovak replica X-ray mirrors for astronomical applications”, SPIE 597, 111-118 (1986).

16 Hudec, R., Valníček, B., Svátek, L., \& Landa, V., "New developments in replica X-ray grazing incidence optics", SPIE 1140, 542-548 (1989).

17 Citterio, O., Conti, G., Mattaini, E., Santambrogio, E., \& Sacco, B., “Optics for X-ray concentrators on board of the astronomy satellite SAX”, SPIE 597, 102-110 (1986).

18 Ulmer, M. P., Purcell, W. R., Jr., Loughlin, J. E. A., \& Kowalski, M. P., "Electroform replication used for multiple X-ray mirror production", ApOpt 23, 4233-4236 (1984).

19 Ulmer, M. P., Matsui, Y., Bedford, D. K., Simnett, G. M., \& Takacs, P. Z., "Electroformed grazing incidence X-ray mirrors for a mirror array telescope", ApOpt 26, 3852-3857 (1987).

20 Citterio, O., Conconi, P., Ghigo, M., Mazzoleni, F., Brauninger, H., Burkert, W., Schultz, N., Gondoin, P., van Katwijk, K., \& Laurance, R., "Results of X-ray measurements on electroformed mirror shells for the XMM project", SPIE 1742, 256-263 (1993).

21 Citterio, O., Conconi, P., Ghigo, M., Mazzoleni, F., Poretti, E., Conti, G., Cusumano, G., Sacco, B., Braeuninger, H. W., \& Burkert, W., "Status of the qualification model of the X-ray optics for the JET-X telescope aboard the Spectrum X-Gamma satellite", SPIE 2515, 44-54 (1995).

22 Romaine, S., Boike, J., Bruni, R., Engelhaupt, D., Gorenstein, P., \& Ramsey, B., "Improved release coatings for electroformed x-ray optics", SPIE 8147, 0W 6pp (2011).

23 Gubarev, M., Ramsey, B., Engelhaupt, D., Kester, T., \& Speegle, C., "Technology development for high-energy xray optics", SPIE 6266, 1I 8pp (2006).

24 Wilson-Hodge, C. A., Gaskin, J., Christe, S., Shih, A., Tennant, A., Swartz, D., Kilaru, K., Elsner, R., Kolodziejczak, J., \& Ramsey, B., "Calibration of the High Energy Replicated Optics to Explore the Sun (HEROES) hard x-ray telescope", JAI 3, 1440007 16pp (2014).

25 Ramsey, B. D., Alexander, C. D., Apple, J. A., Benson, C. M., Dietz, K. L., Elsner, R. F., Engelhaupt, D. E., Ghosh, K. K., Kolodziejczak, J. J., O'Dell, S. L., Speegle, C. O., Swartz, D. A., \& Weisskopf, M. C., "First images from HERO, a hard x-ray focusing telescope", ApJ 568, 432-435 (2002).

26 Ramsey, B. D., Alexander, C. D., Apple, J. A., Benson, C. M., Dietz, K. L., Elsner, R. F., Engelhaupt, D. E., Ghosh, K., Kolodziejczak, J. J., O'Dell, S. L., Speegle, C. O., Swartz, D. A., \& Weisskopf, M. C., "HERO: program status and first images from a balloon-borne focusing hard x-ray telescope", SPIE 4496, 140-145 (2002).

27 Krucker, S., Christe, S., Glesener, L., Ishikawa, S.-n., McBride, S., Glaser, D., Turin, P., Lin, R. P., Gubarev, M., Ramsey, B., Saito, S., Tanaka, Y., Takahashi, T., Watanabe, S., Tanaka, T., Tajima, H., \& Masuda, S., "The Focusing Optics X-ray Solar Imager (FOXSI)", SPIE 8147, 05 14pp (2011).

28 Krucker, S., Christe, S., Glesener, L., Ishikawa, S., Ramsey, B., Gubarev, M., Saito, S., Takahashi, T., Watanabe, S., Tajima, H., Tanaka, T., Turin, P., Glaser, D., Fermin, J., \& Lin, R. P., "The Focusing Optics X-ray Solar Imager (FOXSI): instrument and first flight", SPIE 8862, 0R 12pp (2013).

29 Figueroa-Feliciano, E., Adams, J. S., Baker, R., Bandler, S. R., Dewey, D., Doriese, W. B., Eckart, M. E., Hamersma, R., Hilton, G. C., Hwang, U., Irwin, K. D., Kelley, R. L., Kilbourne, C. A., Kissel, S. E., Leman, S. W., McCammon, D., Oakley, P. H. H., Okajima, T., O'Neal, R. H., Petre, R., Porter, F. S., Reintsema, C. D., Rutherford, J. M., Saab, T., Serlemitsos, P., Soong, Y., Trowbridge, S. N., \& Wikus, P., "Update on the Micro-X sounding rocket payload", SPIE 8443, 1B 17pp (2012).

30 Wikus, P., Adams, J. S., Baker, R., Bandler, S. R., Brys, W., Dewey, D., Doriese, W. B., Eckart, M. E., FigueroaFeliciano, E., Goeke, R., Hamersma, R., Hilton, G. C., Hwang, U., Irwin, K. D., Kelley, R. L., Kilbourne, C. A., Leman, S. W., McCammon, D., Okajima, T., O'Neal, R. H., Jr., Porter, F. S., Reintsema, C. D., Rutherford, J. M., 
Serlemitsos, P., Saab, T., Sato, K., Soong, Y., \& Trowbridge, S. N., "Progress on the Micro-X sounding rocket x-ray telescope: completion of flight hardware", SPIE 7732, 1P 15pp (2010).

31 Pavlinsky, M., Sunyaev, R., Churazov, E., Vikhlinin, A., Sazonov, S., Revnivtsev, M., Arefiev, V., Lapshov, I., Akimov, V., Levin, V., Buntov, M., Semena, N., Grigorovich, S., Babyshkin, V., Predehl, P., Hasinger, G., Böhringer, H., Schmitt, J., Santangelo, A., Schwope, A., \& Wilms, J., "Spectrum-RG astrophysical project", SPIE 7437, 08 11pp (2009).

32 Predehl, P., Andritschke, R., Becker, W., Bornemann, W., Br\&auml;uninger, H., Brunner, H., Boller, T., Burwitz, V., Burkert, W., Clerc, N., Churazov, E., Coutinho, D., Dennerl, K., Eder, J., Emberger, V., Eraerds, T., Freyberg, M. J., Friedrich, P., F\&uuml;rmetz, M., Georgakakis, A., Grossberger, C., Haberl, F., H\&auml;lker, O., Hartner, G., Hasinger, G., Hoelzl, J., Huber, H., von Kienlin, A., Kink, W., Kreykenbohm, I., Lamer, G., Lomakin, I., Lapchov, I., Lovisari, L., Meidinger, N., Merloni, A., Mican, B., Mohr, J., M\&uuml;ller, S., Nandra, K., Pacaud, F., Pavlinsky, M. N., Perinati, E., Pfeffermann, E., Pietschner, D., Reiffers, J., Reiprich, T., Robrade, J., Salvato, M., Santangelo, A. E., Sasaki, M., Scheuerle, H., Schmid, C., Schmitt, J., Schwope, A. D., Sunyaev, R., Tenzer, C., Tiedemann, L., Xu, W., Yaroshenko, V., Walther, S., Wille, M., Wilms, J., \& Zhang, Y.-Y., "eROSITA on SRG", SPIE 9144, 1T 6pp (2014).

33 Meidinger, N., Andritschke, R., Aschauer, F., Bornemann, W., Emberger, V., Eraerds, T., Fürmetz, M., Hälker, O., Hartner, G., Kink, W., Müller, S., Pietschner, D., Predehl, P., Reiffers, J., Walther, S., \& Weidenspointner, G., "Progress of the x-ray CCD camera development for the eROSITA telescope", SPIE 8859, 0B 12pp (2013).

34 Burwitz, V., Predehl, P., Bräuninger, H., Burkert, W., Dennerl, K., Eder, J., Friedrich, P., Fürmetz, M., Grisoni, G., Hartner, G., Marioni, F., Menz, B., Pfeffermann, E., \& Valsecchi, G., "Status of the eROSITA telescope testing and calibrating the x-ray mirror assemblies", SPIE 8861, 0J 10pp (2013).

35 Pavlinsky, M., Akimov, V., Levin, V., Lapshov, I., Tkachenko, A., Semena, N., Buntov, M., Glushenko, A., Arefiev, V., Yaskovich, A., Sunyaev, R., Churazov, E., Gilfanov, M., Grebenev, S., Sazonov, S., Revnivtsev, M., Lutovinov, A., Molkov, S., Kudelin, M., Drozdova, T., Garanin, S., Grigorovich, S., Litvin, D., Lazarchuk, V., Roiz, I., Garin, M., Babyshkin, V., Lomakin, I., Menderov, A., Moskvinov, D., Gubarev, M., Ramsey, B., Kilaru, K., O'Dell, S. L., Kolodziejczak, J., \& Elsner, R., "Status of ART-XC/SRG instrument", SPIE 9144, 1U 11pp (2014).

36 Gubarev, M., Ramsey, B., Elsner, R., O'Dell, S., Kolodziejczak, J., McCracken, J., Zavlin, V., Swartz, D., Kilaru, K., Atkins, C., Pavlinsky, M., Tkachenko, A., \& Lapshov, I., "ART-XC/SRG: status of the X-ray optics development", SPIE 9144, 1V 6pp (2014).

37 Levin, V., Pavlinsky, M., Akimov, V., Kuznetsova, M., Rotin, A., Krivchenko, A., Lapshov, I., \& Oleinikov, V., "ART-XC/SRG: status of the x-ray focal plane detector development", SPIE 9144, 13 9pp (2014).

38 Gubarev, M., Ramsey, B., Kolodziejczak, J. J., O'Dell, S. L., Elsner, R., Zavlin, V., Swartz, D., Pavlinsky, M., Tkachenko, A., \& Lapshov, I., "The calibration of flight mirror modules for the ART-XC instrument on board the SRG mission", SPIE 9144, 4U 6pp (2014).

39 Pivovaroff, M. J., Funk, T., Barber, W. C., Ramsey, B. D., \& Hasegawa, B. H., "Progress of focusing X-ray and gamma-ray optics for small animal imaging", SPIE 5923, 65-78 (2005).

40 Gubarev, M. V., Khaykovich, B., Ramsey, B., Moncton, D., Zavlin, V. E., Kilaru, K., Romaine, S., Rosati, R. E., Bruni, R., Robertson, L., Crow, L., Ambaye, H., \& Lauter, V., "From x-ray telescopes to neutron focusing", SPIE 8147, 0B 8pp (2011).

${ }^{41}$ Khaykovich, B., Gubarev, M. V., Bagdasarova, Y., Ramsey, B. D., \& Moncton, D. E., "From X-ray telescopes to neutron scattering: Using axisymmetric mirrors to focus a neutron beam”, NIMPA 631, 98-104 (2011).

42 Liu, D., Gubarev, M. V., Resta, G., Ramsey, B. D., Moncton, D. E., \& Khaykovich, B., "Axisymmetric grazingincidence focusing optics for small-angle neutron scattering", NIMPA 686, 145-150 (2012).

43 Khaykovich, B., Gubarev, M. V., Zavlin, V. E., Katz, R., Resta, G., Liu, D., Robertson, L., Crow, L., Ramsey, B. D., \& Moncton, D. E., "Novel neutron focusing mirrors for compact neutron sources", PhPro 26, 299-308 (2012).

44 Ablett, J. M., Kao, C. C., \& Lunt, A., "The design and performance of an x-ray micro-focusing system using differentially deposited elliptical mirrors at the National Synchrotron Light Source", RScI 73, 3464-3468 (2002).

45 Ice, G. E., Chung, J.-S., Tischler, J. Z., Lunt, A., \& Assoufid, L., "Elliptical X-ray microprobe mirrors by differential deposition", RScI 71, 2635-2639 (2000). 
46 Cai, Z., Yun, W., \& Plag, P., "Parameter optimization for producing an elliptical surface from a spherical surface by differential deposition", SPIE 2516, 52-68 (1995).

47 Kilaru, K., Ramsey, B. D., Gubarev, M. V., \& Gregory, D. A., "Differential deposition technique for figure corrections in grazing-incidence X-ray optics", OptEn 50, 106501 6pp (2011).

48 Kilaru, K., Ramsey, B. D., Gubarev, M. V., Gaskin, J. A., O'Dell, S. L., \& Zhang, W., "Differential deposition to correct surface figure deviations in astronomical grazing-incidence x-ray optics", SPIE 8147, 0X 8pp (2011).

49 Broadway, D. M., O’Dell, S. L., Ramsey, B. D., \& Weimer, J. J., "Techniques for achieving zero stress in thin films of iridium, chromium, and nickel", SPIE 9510, these proceedings (2015).

50 Broadway, D. M, "Mechanical stress measurement during thin film fabrication", NASA, United States Patent Application \#14,645,994 (2015).

51 Civitani, M. M., Citterio, O., Ghigo, M., Mattaini, E., Pareschi, G., \& Parodi, G., "Thin monolithic glass shells for future high angular resolution and large collecting area x-ray telescope”, SPIE 8884, 1R 11pp (2013).

52 Citterio, O., Civitani, M. M., Pareschi, G., Basso, S., Campana, S., Conconi, P., Ghigo, M., Mattaini, E., Moretti, A., Parodi, G., \& Tagliaferri, G., "Thin fused silica optics for a few arcsec angular resolution and large collecting area X-ray telescope", SPIE 8861, 0V 14pp (2013).

53 Roche, J. M., Gubarev, M. V., Smith, W. S., O'Dell, S. L., Kolodziejczak, J. J., Weisskopf, M. C., Ramsey, B. D., \& Elsner, R. F., "Mounting for fabrication, metrology, and assembly of full-shell grazing-incidence optics", SPIE 9144, 43 9pp (2014). 